\title{
Cost-Effectiveness of Bosutinib for the Treatment of Adult Patients with Chronic Phase Chronic Myeloid Leukemia in the Second-Line Setting
}

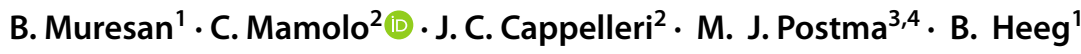

Accepted: 4 June 2021 / Published online: 12 July 2021

(c) The Author(s) 2021

\begin{abstract}
Background A recently conducted matching-adjusted indirect comparison demonstrated that bosutinib improved progressionfree survival, and delayed progression to advanced disease compared with dasatinib and nilotinib in patients with second line (2L) chronic-phase chronic myeloid leukemia (CP-CML). However, the long-term clinical and economic impact of using bosutinib versus dasatinib and nilotinib has not been evaluated. The objective was to determine the cost-effectiveness of bosutinib compared with dasatinib and bosutinib compared with nilotinib from a US payer perspective.

Methods A cost-effectiveness model was developed using partition survival methods and three health states: progressionfree, progression, and death. Trial data (individual patient-level and aggregate-level data) informed the progression-free and overall survival estimates. Costs included drugs and medical resource use. Utility values were obtained from literature. Sensitivity analyses (SAs) included one-way and probabilistic sensitivity analyses (PSAs).

Results Comparing bosutinib versus dasatinib resulted in a gain of 0.4 discounted life years, 1.5 quality-adjusted life years (QALYs), and incremental costs of $\$ 28,459$ (values in 2020 US dollars), for an incremental cost-effectiveness ratio (ICER) of $\$ 19,811$ /QALY gained. Comparing bosutinib versus nilotinib resulted in a gain of 0.8 discounted life-years, 1.8 QALYs, and incremental costs of $\$ 76,563$, for an ICER of $\$ 41,932 / \mathrm{QALY}$ gained. Drug costs and extrapolation distribution type were the main drivers of the model in the one-way SAs. In the PSAs, bosutinib had $>90 \%$ and $>80 \%$ probabilities of being cost-effective at a willingness-to-pay threshold of \$100,000/QALY versus dasatinib and nilotinib, respectively.

Conclusions Our results suggest that compared with dasatinib and nilotinib, bosutinib may represent good value for money for treating $2 \mathrm{~L} \mathrm{CP}-\mathrm{CML}$ patients.
\end{abstract}

\section{Introduction}

Chronic myeloid leukemia (CML) is a type of cancer that affects the blood and bone marrow. In the chronic phase (CP), most patients have few troubling symptoms. However, CML can evolve from a relatively stable disease into a more

B. Heeg

bart.heeg@ingress-health.com

1 Ingress-health Netherlands, Weena 316-318, 3012 NJ Rotterdam, The Netherlands

2 Pfizer Inc, Groton, CT, USA

3 Department of Health Sciences, University Medical Center Groningen, University of Groningen, Groningen, The Netherlands

4 Department of Economics, Econometrics and Finance, Faculty of Economics and Business, University of Groningen, Groningen, The Netherlands rapidly progressing one (accelerated phase; AP), eventually transforming into a rapidly progressing disease (blast phase; BP) [1]. The annual incidence of newly diagnosed cases of CML in the USA is estimated at $1-1.3$ cases per 100,000 . As of 2020, the prevalence of CML is estimated at 112,000. [2] Treatment options depend on the disease phase and other prognostic factors [3].

As some patients will be either refractory or intolerant to their first tyrosine kinase inhibitor (TKI) treatment choice, another TKI can be administered. Available TKIs for second-line (2L) CP-CML include bosutinib, nilotinib, and dasatinib.

Nilotinib is approved in $2 \mathrm{~L}$ for imatinib-resistant or -intolerant Philadelphia chromosome positive $\mathrm{(Ph}+$ ) $\mathrm{CP}$ CML, based on a phase II, single-arm, open-label study [4]. Endpoints of this study included progression-free survival (PFS) and overall survival (OS). Dasatinib is approved for patients with resistance or intolerance to prior therapy, 


\section{Key Points for Decision Makers}

When adjusted for cross-trial differences in disease and patient baseline characteristics, second-generation tyrosine kinase inhibitors (TKIs; bosutinib, dasatinib, and nilotinib) display similar overall survival (OS) and improved progression-free survival (PFS) for bosutinib compared to the other TKIs in second-line treatment (2L) of chronic phase chronic-myeloid leukemia (CPCML).

In a cost-effectiveness model, improved PFS for bosutinib patients translates into a longer time spent in the less expensive progression-free health state (e.g., patients progress slower to the more advanced and expensive stages of the disease) compared to dasatinib and nilotinib patients.

With similar OS (to dasatinib and nilotinib patients), more time spent by the bosutinib patients in the less expensive and with a higher quality of life stage of the disease (progression-free) and less time spent in the more expensive and with a lower quality of life stage of the disease (progression) results in bosutinib being a cost-effective intervention in 2L CP-CML compared to dasatinib and nilotinib.

including imatinib, who have $\mathrm{Ph}+\mathrm{CP}-\mathrm{CML}$. In an openlabel, phase III trial, PFS and OS rates of patients treated with dasatinib were evaluated [5]. Bosutinib is approved for patients newly diagnosed with $\mathrm{Ph}+\mathrm{CP}-\mathrm{CML}$, as well as for patients who no longer benefit from or did not tolerate other treatments. A phase 1/2 study of bosutinib (SKI-606) in Ph+ leukemias included adult 2L CP-CML [6], and assessed OS and PFS.

With no head-to-head trials of bosutinib, dasatinib, and nilotinib in the 2L CP-CML setting, a matching-adjusted indirect comparison (MAIC) comparing OS and PFS was conducted [7]. Analyses showed a statistically significant difference in PFS between bosutinib and nilotinib and between bosutinib and dasatinib, both in favor of bosutinib. No statistically significant differences in OS were observed.

The objective of this study was to assess the cost-effectiveness of bosutinib compared with nilotinib, and bosutinib compared with dasatinib, in terms of OS and PFS, as a $2 \mathrm{~L}$ treatment for CP-CML, using the outcomes of a previously conducted MAIC.

\section{Methods}

The current cost-effectiveness analysis was conducted by means of a standard three-state partitioned survival oncology model [8] from a US-payer perspective that was programmed in Microsoft Excel and Visual Basics for Applications (Fig. 1). This methodology was chosen due to the time-to-event nature of the outcomes (OS, PFS). The model includes mature OS and PFS data for the three TKIs considered (at a follow-up time of 8,6 , and 4 years for bosutinib, dasatinib, and nilotinib, respectively). The effects were measured in quality-adjusted life years (QALYs).

\subsection{Model Structure}

The health states (HS) included in the model are progression-free, progression, and death. Patients enter the model in the progression-free HS (corresponding to the start of their $2 \mathrm{~L}$ of treatment) where they can remain, progress [moving to the third-line of treatment (3L)], or die. Progressed patients can remain in the progression HS or die. Patients progressing to $3 \mathrm{~L}$ treatment cannot be treated with the same TKI as was used in the $2 \mathrm{~L}$ setting. Only bosutinib, nilotinib, dasatinib, and ponatinib are included as possible 3L therapies (other therapies were not included because of their low frequency of use in the 3L setting) [9-11]. The model cycle length is 1 month, and a half-cycle correction was used. This cycle length is a reflection of the clinical expectations, and represents a balance between model computational burden

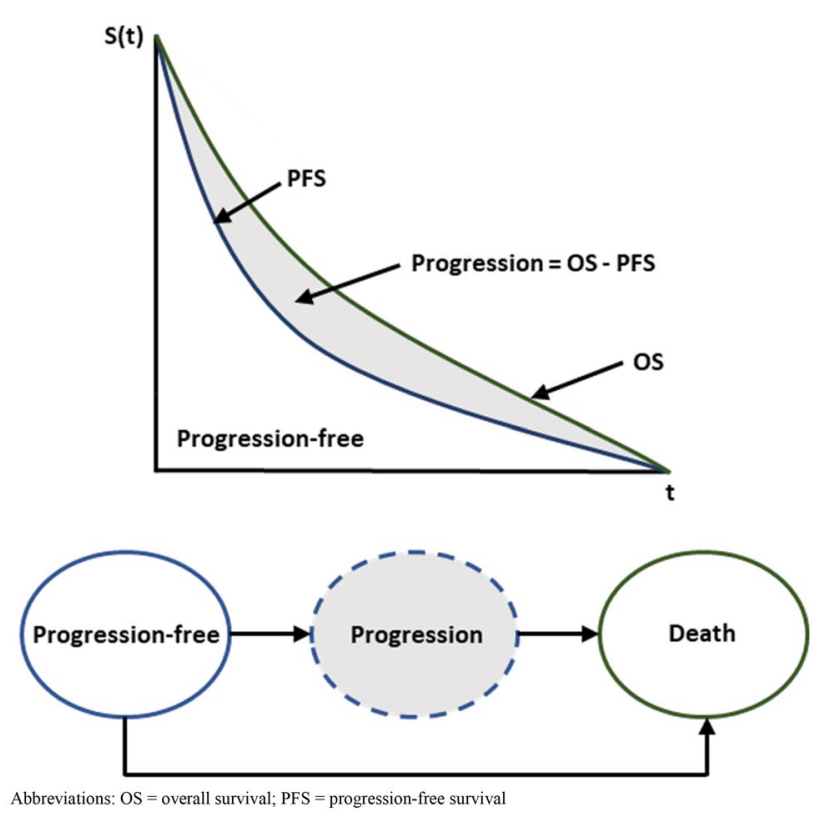

Fig. 1 Health economic model structure. $O S$ overall survival, PFS progression-free survival 
and granularity. Additionally, 1 month is considered short enough to minimize approximation error [12].

Patients entering the model incur treatment costs, face HS-specific utilities, and experience adverse event (AE)related disutilities (decrements in utility) $[13,14]$. To both costs and effects, a 3\% discount rate was applied [15]. All analyses were conducted according to the ISPOR guidelines on good research practices for cost-effectiveness analysis alongside clinical trials $[16,17]$.

\subsection{Model Inputs}

\subsubsection{Clinical Data}

In the absence of head-to-head trials, unanchored MAICs were used to assess the comparative efficacy of nilotinib, dasatinib, and bosutinib [7, 18, 19]. Unanchored MAICs use individual patient data (IPD) from trials of one treatment to match baseline summary statistics reported from trials of another treatment, to adjust for the cross-trial heterogeneity in disease and patient baseline characteristics. After matching, the resulting treatment outcomes are compared across balanced trial populations. For this analysis, only the three second-generation (2G) TKIs (nilotinib, dasatinib, and bosutinib) included in international treatment guidelines for the treatment of 2L CML were considered [20-22]. A critical appraisal of the three clinical trials is available in the Online Supplementary Material (Table S1). Aggregate data were used from the pivotal studies of nilotinib (NCT00109707) [4] and dasatinib (CA180-034) [5], as well as IPD from the bosutinib trial (NCT00261846) [6].

The results of the MAICs showed a statistically significant differentiation in PFS rates between bosutinib and nilotinib with a hazard ratio (HR) of 0.54 (95\% confidence interval [CI] 0.38-0.76) and between bosutinib and dasatinib with a HR of 0.63 (95\% CI 0.44-0.90), both in favor of bosutinib. In terms of OS, the MAIC showed a non-significant HR of 0.82 (95\% CI 0.54-1.26) when dasatinib was compared with bosutinib, and a non-significant HR of 0.72 (95\% CI 0.46-1.13) when nilotinib was compared with bosutinib.

The adjusted OS, PFS Kaplan-Meier (KM) curves, and corresponding hazard ratios (HRs) resulting from the MAIC analysis were implemented in the current health economic model and extrapolated over a life-time horizon. The bosutinib OS and PFS curves used in the model are the ones resulting from the MAIC. Dasatinib and nilotinib OS and PFS curves used in the model are from their corresponding clinical trials, since these are the trials that the bosutinib IPD was matched on in the MAIC. In the base-case scenario, HS transition probabilities of patients were derived from the reweighted trial data from the MAIC, up to the point that it was no longer available. Weibull, lognormal, log-logistic, exponential, and Gompertz parametric survival models were used for the extrapolation of OS and PFS after the point when trial data were no longer available. All parametric survival models were estimated using treatment as a regressor. Goodness of fit was assessed using the Akaike's Information Criterion (AIC) score: the lower the AIC score value, the better the fit.

Adverse event incidence rates were sourced from the US Food and Drug Administration (FDA) labels of each TKI included in the model for non-cardiovascular AEs [23-25], and from the literature [26] for cardiovascular AEs. Adverse event incidence per treatment is assumed to be equal in both the progression-free and the progression HS. The additional mortality caused by cardiovascular AEs is assumed to be indirectly captured by the OS curves from the clinical trials of each comparator. Adverse event incidence rates are shown in Table 1.

\subsubsection{Utility Inputs}

Utilities corresponding to the clinical stages of CML (sourced from Szabo et al. [27], $n=74$ ) were elicited from CML patients in the USA using the time trade-off methodology, assuming seven clinical CML HS. Health-state utilities for progression-free patients are assumed to be equal to the utility of chronic patients who are responsive to treatment (i.e., 0.78). For progressed patients, the utility is calculated as an average between acute and blastic patients with and without response to the treatment (0.395). The values are presented in Table 1. A detailed description of how the utility inputs were derived is shown in Fig. S1 in the Online Supplementary Material.

Adverse event disutilities were sourced from the Tufts cost-effectiveness analysis registry [28], and applied in the model using the additive method described in Ara et al. [29]. The applied input values are presented in Table 1. Disutilities corresponding to acute AEs (e.g., anemia, diarrhea, etc.) were applied only in the first month of treatment, while those corresponding to chronic AEs (e.g., cardiovascular) were assumed to occur with the same incidence throughout the treatment duration. A detailed description of how $\mathrm{AE}$ inputs were derived is shown in Fig. S2 in the Online Supplementary Material.

\subsubsection{Cost Inputs}

Both the intervention and comparators entail drug costs, HS costs, and AE costs. All costs are reported in 2020 US dollars. Drug costs were sourced from the sponsor and consist of the monthly wholesale acquisition costs for January 2017 inflated to February 2020 using annual consumer price index values [30] (Table 1). Drug costs were assumed to be the same for both $2 \mathrm{~L}$ and $3 \mathrm{~L}$ treatment and patients were assumed to be treated until death. 
Table 1 Model inputs with corresponding probabilistic distributions and uncertainty

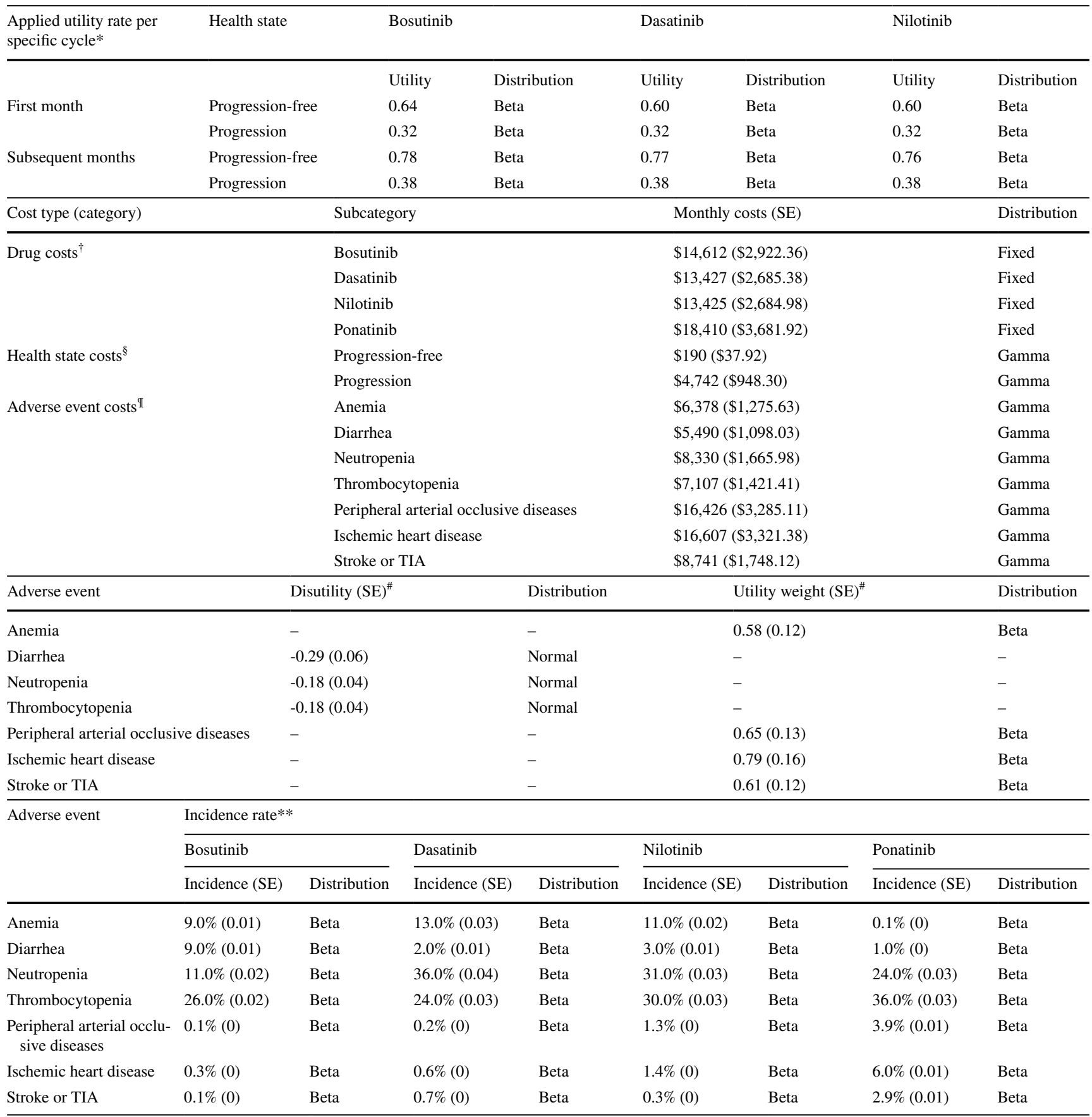

$S E$ standard error, TIA transient ischemic attack

*The utility rates applied in the cost-effectiveness model were obtained from the original utilities (listed in the references) from which adverse event dis-utilities have been subtracted (Fig. S1 in the Online Supplementary Material)

**Adverse event incidence rates were sourced from the corresponding US Food and Drug Administration (FDA) labels for non-cardiovascular adverse events and Chai et al.[26] for cardiovascular adverse events

${ }^{\dagger}$ Drug costs were sourced from the sponsor and represent monthly WACs for January 2017, inflated to February 2020

${ }^{\S}$ Health state costs were sourced from Rochau et al.[31] and from Reed et al.[32] and were inflated to February 2020

${ }^{\mathbb{A}}$ Adverse event costs were calculated based on the International Classification of Diseases Clinical Modification (ICD-CM) codes and the corresponding number of patients discharged from hospital for each adverse event in part

\#Adverse event disutilities/utility weights were derived from the Tufts cost-effectiveness analysis registry 
Health-state costs (sourced from two previous health economic models $[31,32])$ are linked to the clinical stages of CML and are incurred by patients every cycle. For patients in the progression-free HS (linked to the CP-CML stage), only outpatient costs were considered, since typically patients with CP-CML are not admitted as inpatients to hospitals as a result of their diagnosis. For patients in the progression HS (linked to the AP-CML and BP-CML stages), both inpatient and outpatient costs were considered, since patients who are initially treated as outpatients may be admitted to hospitals.

Adverse event costs were calculated based on the International Classification of Diseases Clinical Modification codes and the corresponding number of patients discharged from hospital for each AE in part (Table 1).

\subsection{Deterministic Analyses}

In the base-case analysis, MAIC-reweighted OS and PFS $\mathrm{KM}$ curves were applied in the model for nilotinib and dasatinib and corresponding IPD-derived KM curves for bosutinib, respectively. Parametric curve extrapolation started from the cut-off point of the trial data using the best fitting model, according to the AIC scores. Treatment-specific outcomes were estimated for costs, life years (LYs), and QALYs gained. Incremental values for the same parameters were generated, along with the incremental cost-effectiveness ratio (ICER).

\subsection{Sensitivity Analyses}

Uncertainty around the results was explored in a one-way sensitivity analysis (SA). All model input parameters were varied, one at a time, while holding the remainder of the parameters constant. One-way SAs have been performed by applying the upper and lower boundary given by the distribution around each of the parameters in the model (with a 95\% CI) (Table 1). Where distributions were not available, a $\pm 20 \%$ range from the point estimate was assumed. The result is graphically illustrated through a tornado diagram, showing the ten parameters having the highest impact on the ICER.

\subsection{Probabilistic Analyses}

A probabilistic SA (PSA) was performed to examine the combined effect of the uncertainty surrounding all variable parameters of the model. Values were sampled from the uncertainty distributions specific for each parameter (Table 1). Parameter-specific uncertainty distribution was estimated based on the standard error (SE) of the deterministic point estimate. In the absence of the latter, a $20 \%$ SE was assumed. The PSA performed on the deterministic point estimates included 1,000 simulations and its results are presented in a cost-effectiveness plane and in the costeffectiveness acceptability curve (CEAC) [33].

\subsection{Scenario Analyses}

Several scenarios were simulated to explore uncertainty around the structural assumptions used in the model, and their resulting ICERs were compared. In a first-scenario analysis (ScA1), alternative parametric survival curve extrapolations were tested on OS and PFS KM data. Cost, utility, and adverse event input parameters were kept constant across the simulated scenarios. In a second-scenario analysis (ScA2), all treatment costs were set to zero to better assess the ICER in the absence of treatment cost uncertainty.

\section{Results}

\subsection{Base-Case Analysis}

\subsubsection{Deterministic Results}

According to the AIC score, log-normal was the best fitting model to OS and PFS KM data in both comparisons and therefore was applied in the base-case scenario. Survival extrapolations of the best fitting models as well as the AIC scores corresponding to all models are shown in Fig. S3a-d and in Table S2 in the Online Supplementary Material.

In the comparison of bosutinib with dasatinib, 0.4 and 1.5 discounted incremental LYs and QALYs were gained, respectively, with bosutinib treatment. The incremental total costs were $\$ 28,459$, with a discounted ICER of $\$ 19,811$ per QALY gained. In the comparison of bosutinib with nilotinib, 0.8 and 1.8 discounted incremental LYs and QALYs were gained, respectively, with bosutinib treatment. The incremental total costs were $\$ 76,563$, with a discounted ICER of \$41,932 per QALY gained. The gain in LYs and QALYs largely occurs in the progression-free HS. The additional costs are divided over the progression-free and the progression HS (representing $87 \%$ and $13 \%$ of the total time, respectively, in the comparison with dasatinib) and over the progression-free and the progression HS (representing $88 \%$ and $12 \%$, respectively, in the comparison with nilotinib). In both comparisons, the benefit for bosutinib is derived from the higher utility gained in the progression-free HS compared to the lower utility gained in the progression HS for dasatinib and nilotinib.

The higher PFS for patients on bosutinib compared to patients on dasatinib translates into more LYs and QALYs gained in the less costly progression-free HS than in the more expensive progression HS. Table 2 shows the total and disaggregated costs for each HS, total QALYs gained, 
Table 2 Discounted outcomes of the comparison between bosutinib, dasatinib, and nilotinib

\begin{tabular}{|c|c|c|c|c|c|c|c|c|c|}
\hline TKI & Total costs & Total LY & \multicolumn{2}{|c|}{ Total QALYs } & Incremental costs & $\begin{array}{l}\text { Incremental } \\
\text { QALYs }\end{array}$ & \multicolumn{2}{|c|}{ ICER (costs/QALY) } & $\mathrm{NMB}^{\mathrm{a}}$ \\
\hline Bosutinib & $\$ 2,376,024$ & 12.9 & 9.5 & \multicolumn{2}{|r|}{$\$ 28,459$} & 1.5 & \multicolumn{2}{|c|}{$\$ 19,811$} & $\$(1,426,024)$ \\
\hline Dasatinib & $\$ 2,347,565$ & 12.5 & 8.0 & & & & & & $\$(1,547,565)$ \\
\hline Bosutinib & $\$ 2,316,965$ & 12.6 & 9.3 & \multicolumn{2}{|r|}{$\$ 76,563$} & 1.8 & \multicolumn{2}{|c|}{$\$ 41,932$} & $\$(1,386,965)$ \\
\hline Nilotinib & $\$ 2,240,402$ & 11.8 & 7.5 & & & & & & $\$(1,490,402)$ \\
\hline \multirow[t]{2}{*}{ Health state } & \multicolumn{3}{|l|}{ Bosutinib } & \multicolumn{3}{|c|}{ Dasatinib } & \multicolumn{3}{|c|}{ Increment } \\
\hline & LYs & QALYs & Costs & LYs & QALYs & Costs & LYs & QALYs & Costs \\
\hline $\begin{array}{l}\text { Progression- } \\
\text { free }\end{array}$ & 11.6 & 9.0 & $\$ 2,053,822$ & 8.5 & 6.5 & $\$ 1,383,252$ & 3.1 & 2.5 & $\$ 670,570$ \\
\hline Progression & 1.3 & 0.5 & $\$ 314,640$ & 4.0 & 1.5 & $\$ 954,728$ & -2.7 & -1 & $\$(640,088)$ \\
\hline \multirow[t]{2}{*}{ Health state } & \multicolumn{3}{|l|}{ Bosutinib } & \multicolumn{3}{|c|}{ Nilotinib } & \multicolumn{3}{|c|}{ Increment } \\
\hline & LYs & QALYs & Costs & LYs & QALYs & Costs & LYs & QALYs & Costs \\
\hline $\begin{array}{l}\text { Progression- } \\
\text { free }\end{array}$ & 11.4 & 8.8 & $\$ 2,021,919$ & 7.8 & 6.0 & $\$ 1,278,879$ & 3.6 & 2.8 & $\$ 743,040$ \\
\hline Progression & 1.2 & 0.4 & $\$ 287,439$ & 4.0 & 1.5 & $\$ 951,646$ & -2.8 & -4.1 & $\$(664,207)$ \\
\hline
\end{tabular}

$T K I$ tyrosine kinase inhibitor, $L Y$ life years, $Q A L Y$ quality-adjusted life years, ICER incremental cost-effectiveness ratio, $N M B$ net monetary benefit (at the $\$ 100,000$ willingness-to-pay threshold)

${ }^{a}$ Net monetary benefit was calculated for each therapy as: effectiveness (QALYs) * $\$ 100,000$ (willingness-to-pay threshold) minus the total costs

as well as the incremental costs and QALYs for each comparison.

\subsection{Sensitivity Analysis}

The sensitivity analysis (SA) showed that drug costs have the highest impact on the ICER. High drug costs for bosutinib have a positive impact on the ICER, whereas high drug costs for dasatinib and nilotinib have a negative effect on the ICER. The ICER also showed increased sensitivity to the distribution of the OS and PFS extrapolation models. This was captured through the inclusion of the log-normal intercept in the top ten parameters having the highest impact on the incremental estimates. The results of the SA are displayed in the tornado diagrams shown in Fig. 2a, b.

\subsection{Probabilistic Results}

The results of the PSA following 1,000 iterations are shown in Fig. 3a, b. In the comparison with dasatinib, the ICER distribution is relatively compact, with most of the values between negative $\$ 200,000$ and $\$ 200,000$. In the comparison with nilotinib, the ICER distribution is sparser (therefore displaying more uncertainty), with most of the values between negative $\$ 200,000$ and $\$ 400,000$. According to the cost-effectiveness acceptability curves (Fig. $4 a, b$ ), the probability that bosutinib is a cost-effective option compared with dasatinib at the $\$ 100,000$ threshold is estimated to be $>$ $90 \%$. The probability that bosutinib is a cost-effective option compared to nilotinib at the same threshold is estimated to be $>80 \%$, thus confirming the higher uncertainty than in the bosutinib versus dasatinib comparison.

\subsection{Scenario Analyses}

The SA results showed evidence of high sensitivity of the ICER to the parametric survival models used to extrapolate OS and PFS for the three TKIs being compared. The first scenario analysis displays the comparative outcomes between the different extrapolation scenarios and the base case for the comparison between bosutinib and dasatinib and between bosutinib and nilotinib, respectively.

Compared to the base case, the first scenario showed higher incremental QALYs when other parametric curves are used for extrapolation than those from the base case (lognormal). Although the base case seems a more conservative approach, the additional QALYs gained in the alternative scenarios proved to be more costly than the ones gained in the base case. Because of that, the ICER was also higher when alternative extrapolation curves were used.

In the second scenario, when all treatment costs were set to zero (to exclude treatment cost-related uncertainty), bosutinib patients still had higher overall LY and QALY gains, resulting in dominant ICERs. 
a

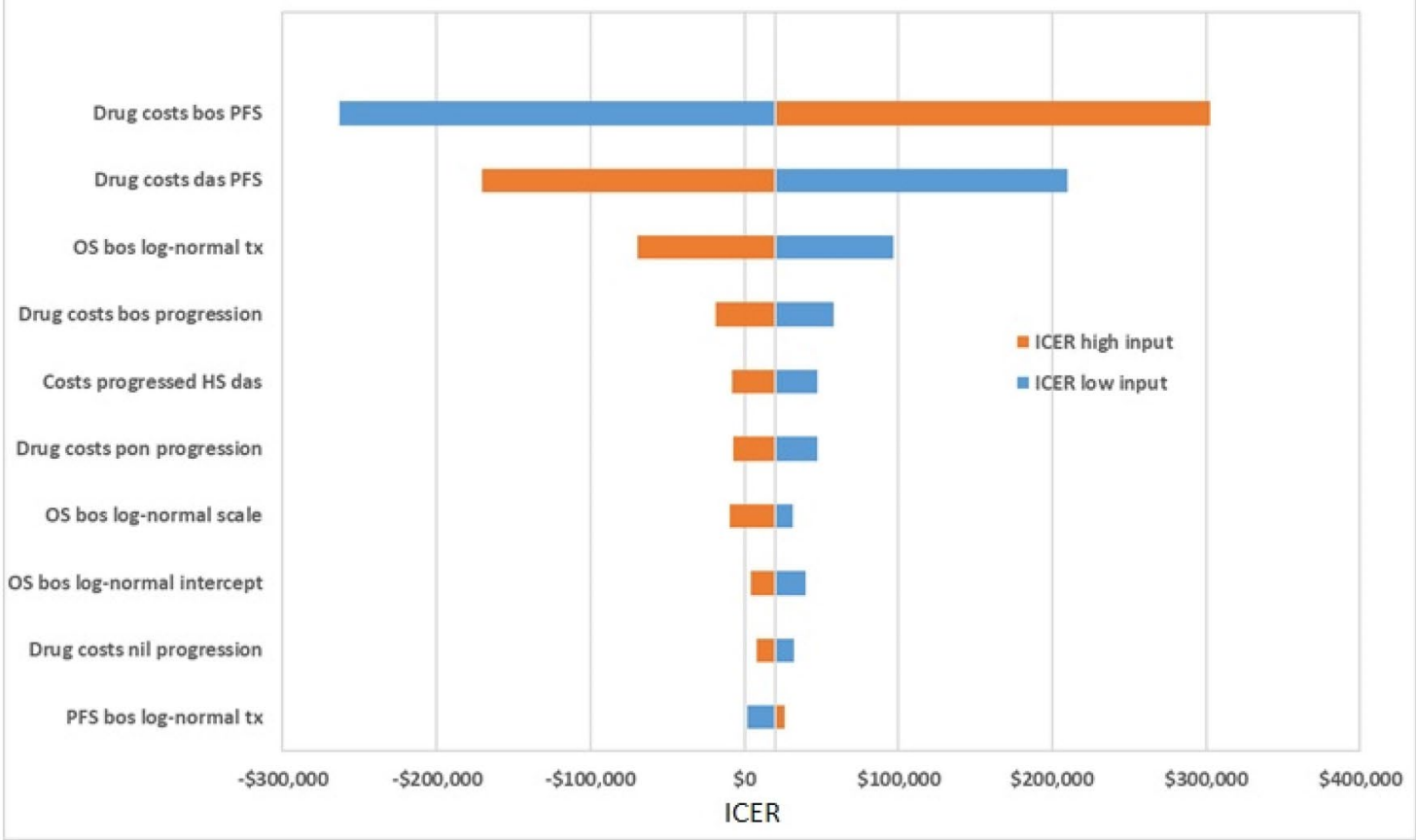

Abbreviations: bos = bosutinib; PFS = progression-free survival; das = dasatinib; OS = overall survival; $t \mathrm{t}=$ treatment; pon = ponatinib; HS = health state; ICER - incremental cost-effectiveness ratio

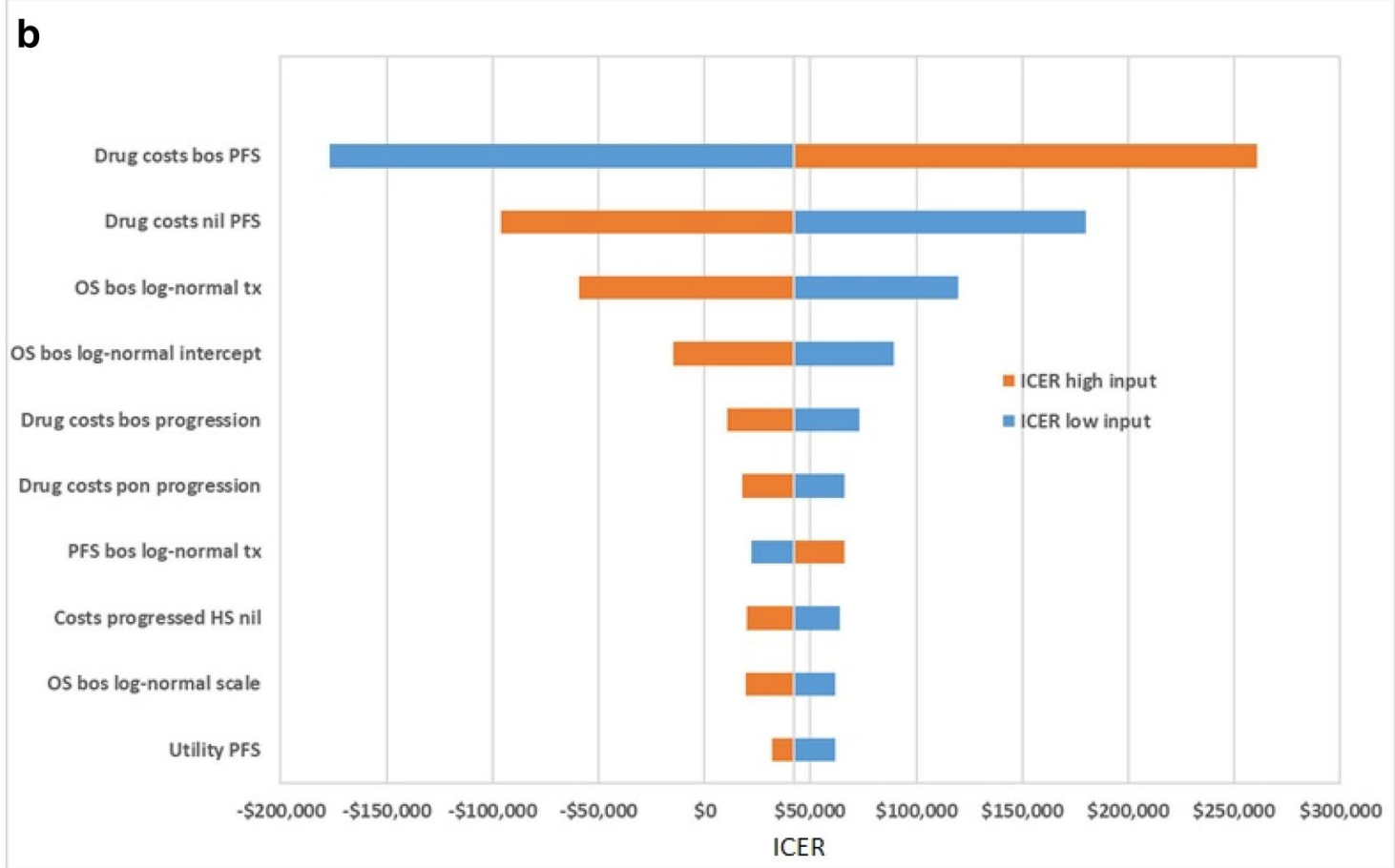

Abbreviations: bos = bosutinib; PFS = progression-free survival; nil = nilotinib; OS = overall survival; tx = treatment; pon = ponatinib; HS = health state; ICER - incremental cost-effectiveness ratio

Fig. 2 a Deterministic sensitivity analysis outcomes: tornado diagrams for the comparison between bosutinib and dasatinib. bos bosutinib, $P F S$ progression-free survival, nil nilotinib, $O S$ overall survival, $t x$ treatment, pon ponatinib, $H S$ health state, ICER incremental costeffectiveness ratio. b Deterministic sensitivity analysis outcomes: tor- nado diagrams for the comparisons between bosutinib and nilotinib. bos bosutinib, $P F S$ progression-free survival, nil nilotinib, $O S$ overall survival, $t x$ treatment, pon ponatinib, $H S$ health state, ICER incremental cost-effectiveness ratio 


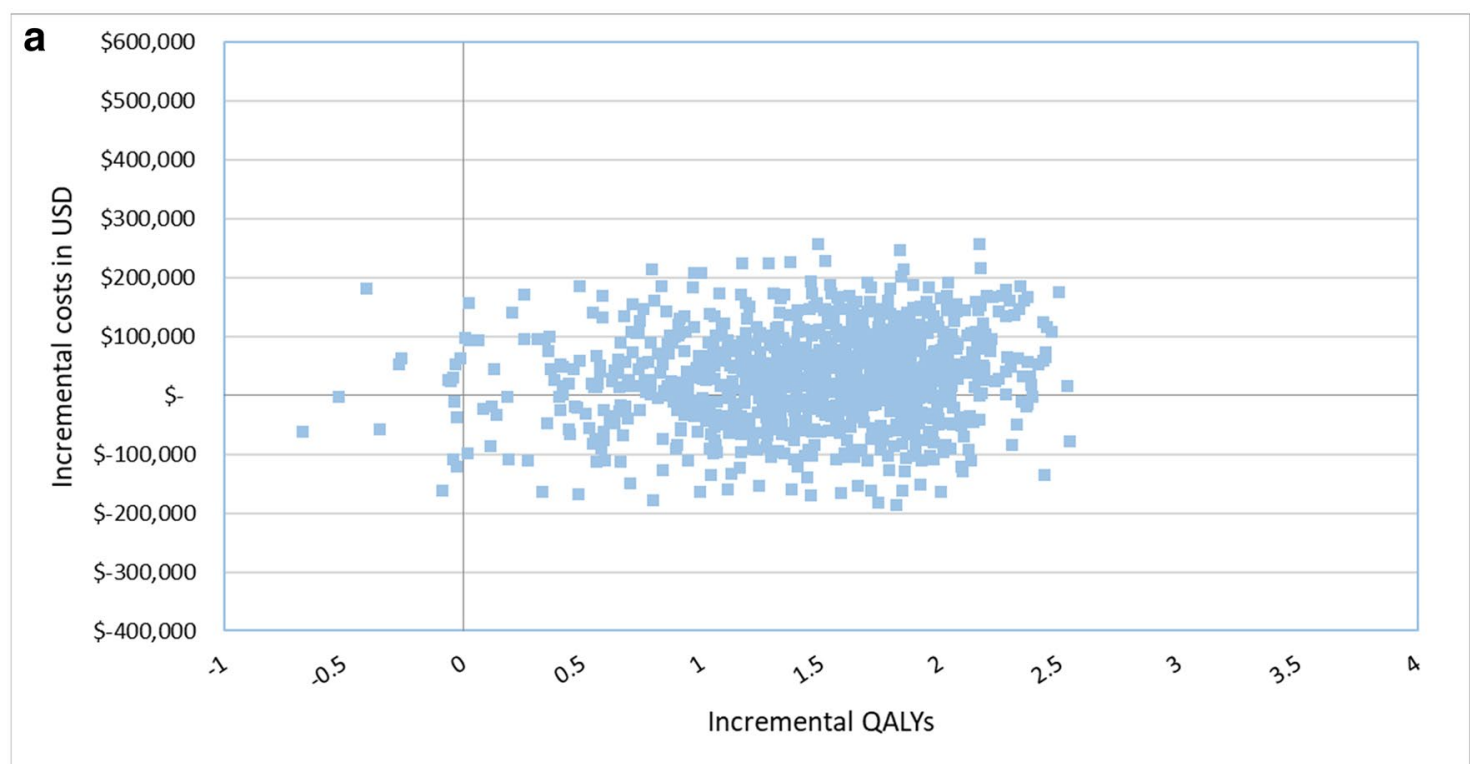

Abbreviations: USD - United States dollars; QALYs = quality-adjusted life years

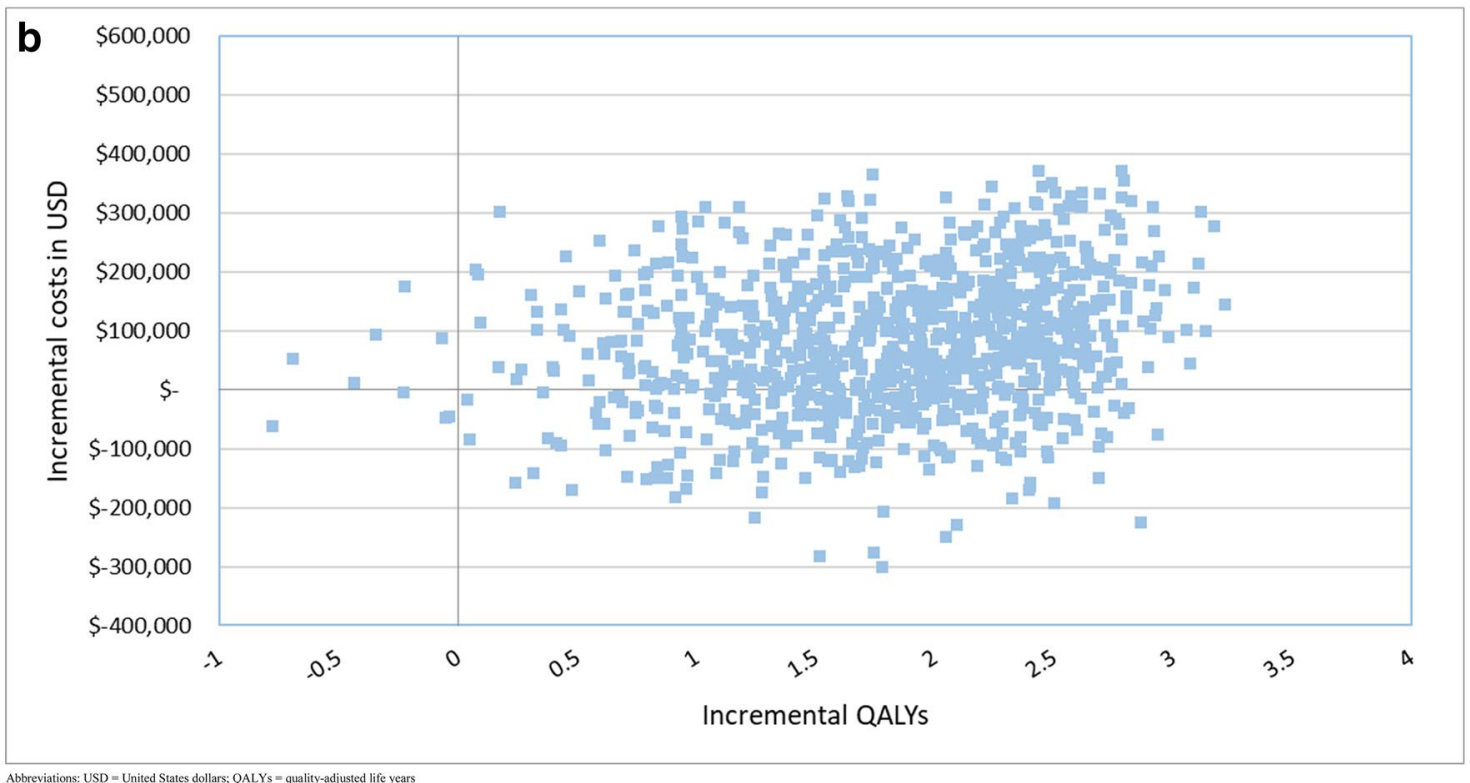

Fig. 3 a Cost-effectiveness plane for the comparison between bosutinib and dasatinib. USD United States dollars, QALYs quality-adjusted life years. b Cost-effectiveness plane for the comparison between

Table S3 in the Online Supplementary Material shows the comparative ICERs between the two alternative scenarios and the base case.

\section{Discussion}

The purpose of this analysis was to compare the cost-effectiveness of available TKI therapies in 2L CP-CML. bosutinib and nilotinib. USD United States dollars, QALYs qualityadjusted life years

A previously conducted MAIC [7] showed that in terms of OS, findings were numerically in favor of bosutinib although not statistically significant when compared with dasatinib and nilotinib and statistically significant in favor of bosutinib for PFS, relative to the same two TKIs. Subsequently, we developed a health economic model centered on OS and PFS, to capture the potential benefit demonstrated by the MAIC analyses. Following the latter analyses, the resulting OS and PFS KM curves were extrapolated using 


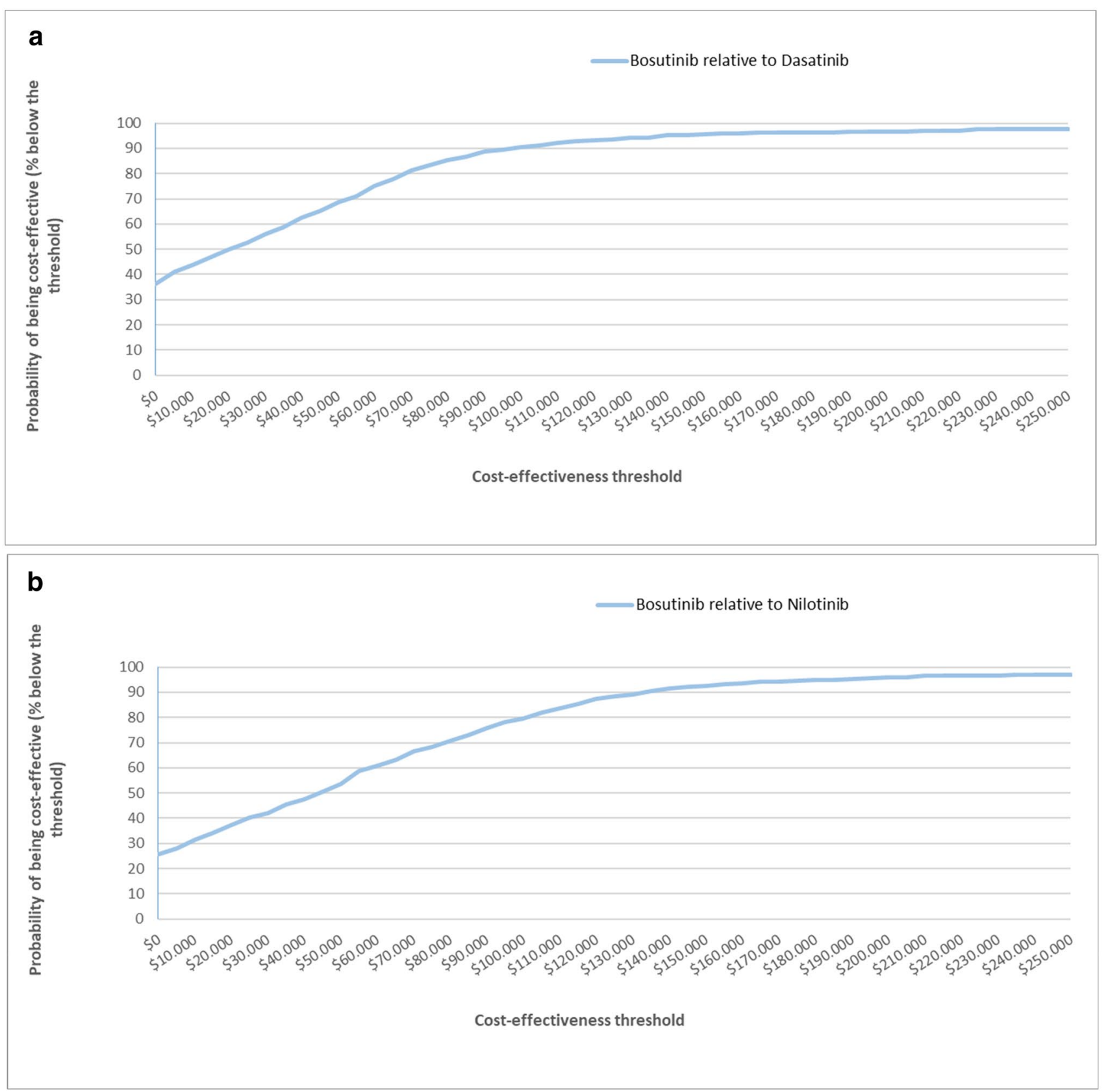

Fig. 4 a Cost-effectiveness acceptability curve for the comparison between bosutinib and dasatinib. b Cost-effectiveness acceptability curve for the comparison between bosutinib and nilotinib

parametric survival models and the extrapolations incorporated into the cost-effectiveness model. Dasatinib and nilotinib were included as comparators in the model in the $2 \mathrm{~L}$ treatment setting. Ponatinib was included as one of the $3 \mathrm{~L}$ therapies for patients who progressed [34].

The model results indicate that bosutinib is cost-effective relative to dasatinib and nilotinib. For the base case, when compared with dasatinib, the model showed a discounted ICER of $\$ 19,811$ per QALY gained (1.5 QALYs gained at a cost of $\$ 28,459)$. Although patients on bosutinib incurred slightly higher costs than patients on dasatinib, those on bosutinib also gained overall more QALYs relative to the patients on dasatinib. Patients on dasatinib gained more QALYs in the (more expensive) progression HS, while patients on bosutinib gained more QALYs in the (less expensive) progression-free HS. Because the additional QALYs gained in progression by patients on dasatinib do not outweigh the additional QALYs gained by the patients on bosutinib in the progression-free HS, bosutinib appeared to be more cost-effective than dasatinib. 
When compared with nilotinib, the model showed a discounted ICER of $\$ 41,932$ per QALY gained (1.8 QALYs gained at a cost of \$76,563). Similar to the comparison with dasatinib, patients on bosutinib incur slightly higher costs than patients on nilotinib, however they also gain more QALYs overall. Bosutinib is more cost-saving than nilotinib due to the fewer QALYs gained in the more expensive progression HS. Additionally, patients on bosutinib gain more QALYs in the less expensive progression-free HS than patients on nilotinib. For these reasons, bosutinib appears more cost-effective than nilotinib.

The underlying evidence suggests that a differentiation in terms of OS between the three TKIs is unlikely, however patients on bosutinib benefit from an improved PFS compared to patients receiving other TKIs. This results into the cost-effectiveness of bosutinib.

A SA and a PSA assessed the uncertainty surrounding the model parameters. The CEACs displayed a probability of $>90 \%$ and approximately $80 \%$ of bosutinib being costeffective against dasatinib and nilotinib, respectively, at the $\$ 100,000$ threshold. The ICER tornado diagrams showed in both cases that drug costs are the input values to which the ICER is most sensitive, followed by the parametric extrapolation models.

Scenario analysis showed that OS and PFS benefits were sensitive to the parametric approach considered. The lognormal model had the lowest AIC score and the best visual fit to the data for both comparisons and hence was used in the base case [35]. Other parametric extrapolations increased the incremental survival (LY), resulting in higher ICER values, due to the longer time patients spend in the more expensive progression HS. The generalized gamma extrapolation model [35] typically generates convergence issues when run in R/Stata and hence was not used in this cost-effectiveness model. Another scenario analysis showed that when drug costs are excluded from the analysis, dominant ICERs result, due to the higher LY and QALYs gains for bosutinib patients compared to dasatinib and nilotinib patients.

There is no strict threshold value for the willingness-topay (WTP) per QALY gained in the USA; however, most submissions aim for a maximum of $\$ 150,000 /$ QALY [36]. Health interventions showing ICERs $<\$ 50,000 /$ QALY are regarded of high value; ICERs between \$50,000/QALY and $\$ 150,000 /$ QALY are considered of intermediate value, while those with ICERs $>\$ 150,000 /$ QALY are seen as low value [37]. Our analyses have shown that, compared with dasatinib and nilotinib, bosutinib is a highly cost-effective intervention at the $\$ 50,000 / \mathrm{QALY}$ threshold. However, our model results were very sensitive to a range of extrapolation models used for OS and PFS, leading to much higher ICERs in the scenario analysis. Even with ICERs up to five times higher than those in the base case, bosutinib seems to be a cost-effective intervention at the higher WTP thresholds $(\$ 150,000)$ applied in practice by US payers. Therefore, from the wider ICER-acceptance perspective, the conclusions of the current cost-effectiveness model are stable across all parametric distributions considered.

$\mathrm{CP}-\mathrm{CML}$ is a rare indication, with limited data to validate. Nevertheless, the MAIC underlying the model was validated with clinicians. The HRs underlying the model are statistically significant for PFS but not for OS. Since PFS is regarded only as an intermediate endpoint in some countries, the generalizability of the model results depends on the uncertainty surrounding OS. In the absence of longterm OS data for the therapies considered in the paper, the extrapolations rely on statistical fit. The uncertainty around OS extrapolations has been acknowledged and quantified in a SA and PSA: different parametric models do not seem to impact the conclusions of this paper.

Previous studies have examined the cost-effectiveness of nilotinib and dasatinib. Using a lifetime Markov disease progression and cost-effectiveness model, Ward et al. [38] concluded that dasatinib is dominant in the UK compared to nilotinib in the first-line and the 2L CML setting, due to improved mean survival outcomes and lower acquisition costs (savings of $£ 29,308$ and $£ 28,706$ respectively). A Markov model published in Wu et al. [39] showed a dominance of dasatinib over nilotinib, with the findings of higher health gains (6.34 compared to 6.19 QALYs) at lower costs (\$133,866 and \$141,184, respectively). Finally, Hoyle et al. [40] concluded using an area-under-the-curve partitioned survival model that although patients on dasatinib have higher health gains than patients on nilotinib (7.85 compared to 7.63 QALYs gained), the associated costs are higher for dasatinib than for nilotinib (£161,432 and $£ 70,143$, respectively). At the time when the cost-effectiveness analysis was conducted, bosutinib was not available (hence was not considered), and the OS data were immature. Additional information on the current model validation (assumptions and results, using the validation-assessment tool published in Vemer et al. [41]) is available in the Online Supplementary Material.

Limitations of the previously conducted MAIC [7] apply to the current health economic model as well, since the latter is based on the former. Access restricted to marginal covariates means that only observed differences between the trials could be corrected for (e.g., baseline characteristics that were reported in the published trials). By matching the baseline characteristics of the bosutinib patients with those of the nilotinib/dasatinib patients, it is implicitly assumed that the latter patients represent the target population. For a more objective comparison, the target population should be the one in national cohorts or CP-CML registry studies [19]. Another limitation is the general MAIC design allowing only for one-by-one comparisons that generate survival input parameters separately for the bosutinib versus dasatinib and 
bosutinib versus nilotinib comparisons. This translates into it being impossible to assess the cost-effectiveness of dasatinib versus nilotinib.

Additionally, the model does not consider more granular CML HS, such as AP and BP over time, since a differentiation between $\mathrm{AP}$ and $\mathrm{BP}$ was not relevant for modeling the treatment pathway. Although clinically BP is a more serious condition than AP, it is progressing out of the chronic phase that represents the critical change in a patient's health status. For patients progressing to AP/BP, different utilities have been assigned, depending on whether patients had a response to the treatment or not. However, since the exact number of patients progressing to $\mathrm{AP} / \mathrm{BP}$ with/without response is not reported for nilotinib and dasatinib, equal weights per each sub-state in the progression HS were assumed and an average between them was included in the model. Cardiovascular AEs considered in this model could potentially be fatal and hence lead to a conflation with OS. Chai et al. [26] do not report the number of patients that survived the event, and therefore an assumption was made that all cardiovascular AEs are non-fatal. Nevertheless, AEs do not seem to impact the ICER.

Another limitation is that patients in the model can only switch to another treatment if they progress; however, in the real-world setting, patients may switch to other therapies for more various reasons, which were not incorporated in the current model, such as intolerance and the availability of the newer therapies.

In conclusion, our analyses demonstrate that bosutinib seems to be cost-effective relative to dasatinib and nilotinib, with discounted base-case ICERs of $\$ 19,811$ and $\$ 41,932$, respectively.

Supplementary Information The online version contains supplementary material available at https://doi.org/10.1007/s40258-021-00666-0.

\section{Declarations}

Funding This study was sponsored by Pfizer Inc.

Conflict of Interest C. Mamolo and J.C. Cappelleri are employees and shareholders of Pfizer Inc. B. Muresan and B. Heeg are employees of Ingress-Health $\mathrm{BV}$, and were paid consultants to Pfizer in connection with the development of this article, which provides consulting and other research services to pharmaceutical, medical device, and related organizations. In their salaried positions, they work with a variety of companies and organizations, and are precluded from receiving payment or honoraria directly from these organizations for services rendered. B. Heeg is also an equity holder of Ingress-Health. M.J. Postma received grants and honoraria from various pharmaceutical companies, including those developing, producing, and marketing the drugs included in this paper. Also, M.J. Postma is advisor to Asc Academics and holds stocks in Pharmacoeconomics Advice Groningen, HealthEcore and Ingress-Health; the latter company was paid by Pfizer Inc. to conduct this study and develop this article.
Ethics Approval Not applicable.

Consent to Participate Not applicable.

Code Availability Visual Basic code used for PSA and DSA programming is available on request.

Author Contribution Conception and design of the work-BM, CM, $\mathrm{JCC}, \mathrm{BH}$; acquisition and analysis of the data-BM, CM, JCC, BH; interpretation of the data- $\mathrm{BM}, \mathrm{CM}, \mathrm{JCC}, \mathrm{MJP}, \mathrm{BH}$; drafting the work and revising it for important intellectual content-BM, CM, JCC, MJP, $\mathrm{BH}$; final approvals for publication and agreement to be accountable for all aspects of the work-BM, CM, JCC, MJP, BH.

Availability of Data and Material Available on request (stored on password-protected external hard drives).

Open Access This article is licensed under a Creative Commons Attribution-NonCommercial 4.0 International License, which permits any non-commercial use, sharing, adaptation, distribution and reproduction in any medium or format, as long as you give appropriate credit to the original author(s) and the source, provide a link to the Creative Commons licence, and indicate if changes were made. The images or other third party material in this article are included in the article's Creative Commons licence, unless indicated otherwise in a credit line to the material. If material is not included in the article's Creative Commons licence and your intended use is not permitted by statutory regulation or exceeds the permitted use, you will need to obtain permission directly from the copyright holder. To view a copy of this licence, visit http://creativecommons.org/licenses/by-nc/4.0/.

\section{References}

1. Leukaemia Foundation Australia website. Chronic myeloid leukemia. 2020. https://www.leukaemia.org.au/disease-information/ leukaemias/chronic-myeloid-leukaemia/. Accessed 23rd Mar.

2. Huang X, Cortes J, Kantarjian H. Estimations of the increasing prevalence and PLateau prevalence of chronic myeloid leukemia in the era of tyrosine kinase inhibitors therapy. Cancer. 2012;118(12):3123-7.

3. American Cancer Society website. Treating chronic myeloid leukemia by phase. 2020. https://www.cancer.org/cancer/chron ic-myeloid-leukemia/treating/treating-by-phase.html. Accessed 23rd Mar 2020.

4. Kantarjian HM, Giles FJ, Bhalla KN, et al. Nilotinib is effective in patients with chronic myeloid leukemia in chronic phase after imatinib resistance or intolerance: 24-month follow-up results. Blood. 2011;117(4):1141-5.

5. Shah NP, Guilhot F, Cortes JE, et al. Long-term outcome with dasatinib after imatinib failure in chronic-phase chronic myeloid leukemia: follow-up of a phase 3 study. Blood. 2014;123(15):2317-24.

6. Cortes JE, Kantarjian HM, Brümmendorf TH, et al. Safety and efficacy of bosutinib (SKI-606) in chronic phase Philadelphia chromosom-positive chronic myeloid leukemia patients with resistance or intolerance to imatinib. Blood. 2011;118(17):4567-76.

7. Cortes JE, Muresan B, Mamolo C, et al. Matching-adjusted indirect comparison of bosutinib, dasatinib and nilotinib effect on survival and major cytogenetic response in treatment of secondline chronic phase myeloid leukemia. Curr Med Res Opin. 2019; 35(9). 
8. University of York Glossary. Partitioned Survival Model. 2019. https://yhec.co.uk/glossary/partitioned-survival-model/. Accessed 4th Dec 2019.

9. García-Gutiérrez V, Hernández-Boluda JC. Current treatment options for chronic myeloid leukemia patients failing secondgeneration tyrosine kinase inhibitors. J Clin Med. 2020;9(7):2251.

10. Ongoren S, Eskazan AE, et al. Third-line treatment with secondgeneration tyrosine kinase inhibitors (dasatinib or nilotinib) in patients with chronic myeloid leukemia after two prior TKIs: reallife data on a single center experience along with the review of the literature. Hematology. 2018;23(4):212-20.

11. Hochhaus A, Breccia M, et al. Expert opinion - management of chronic myeloid leukemia after resistance to second-generation tyrosine kinase inhibitors. Leukemia. 2020;34:1495-502.

12. O'Mahony JF, Newall AT, et al. Dealing with time in health economic evaluation: methodological issues and reccomendations for practice. Pharmacoeconomics. 2015;33(12):1255-68.

13. Whitehead SJ, Ali S. Health outcomes in economic evaluation: the QALY and utilities. Br Med Bull. 2010; 96(1) (December 2010).

14. Disutility [online]. York; York Health Economics Consortium; 2016. https://yhec.co.uk/glossary/disutility/. Accessed Jan 2020.

15. Haacker M, Hallett TB, et al. On discount rates for economic evaluations in global health. Health Policy Plan. 2020;35(1):107-14.

16. Ramsey S, Wilke R, Briggs A, et al. Good research practices for cost-effectiveness analysis alongside clinical trials: the ISPOR RCT-CEA Task Force Report. Value Health. 2005;8(5).

17. Ramsey SD, Wilke RJ, Glick H, et al. Cost-effectiveness analysis alongside clinical trials II-an ISPOR good research practices task force report. Value Health. 2015;18:161-72.

18. Signorovitch JE, Sikirica V, Erder MH, et al. Matching-adjusted indirect comparisons: a new tool for timely comparative effectiveness research. Value Health. 2012;15(6):940-7.

19. Phillippo DM, Ades AE, Dias S, et al. Methods for populationadjusted indirect comparisons in health technology appraisal. Med Decis Making. 2018;38(2):200-11.

20. Hochhaus A, Baccarani M, et al. European LeukemiaNet 2020 recommendations for treating chronic myeloid leukemia. Leukemia. 2020;34:996-984

21. Baccarani M, Castagnetti $\mathrm{F}$, et al. Treatment recommendations for chronic myeloid leukemia. Mediterr J Hematol Infect Dis. 2014;6(1):e2014005.

22. Hochhaus A, Saussele A, et al. Chronic myeloid leukaemia: ESMO Clinical Practice Guidelines for diagnosis, treatment and follow-up. Ann Oncol. 2017;28(Supplement 4):iv41-51.

23. Food and Drug Administration, USA. Highlights of prescribing information for bosutinib (FDA label). 2020. https://www. accessdata.fda.gov/drugsatfda_docs/label/2012/203341lbl.pdf. Accessed Jan 2020.

24. Food and Drug Administration, USA. Highlights of prescribing information for nilotinib (FDA label). 2020. https://www.acces sdata.fda.gov/drugsatfda_docs/label/2010/022068s004s005lbl. pdf. Accessed Jan 2020.

25. Food and Drug Administration, USA. Highlights of prescribing information for dasatinib (FDA label). 2020. https://www.acces sdata.fda.gov/drugsatfda_docs/label/2017/021986s0201bl.pdf. Accessed Jan 2020
26. Chai-Adisaksopha C, Lam W, Hillis C. Major arterial events in patients with chronic myeloid leukemia treated with tyrosine kinase inhibitors: a meta-analysis. Leuk Lymphoma. 2016;57(6):1300-10.

27. Szabo SM, Levy AR, Davis C, Holyoake TL, Cortes J. A multinational study of health state preference values associated with chronic myelogenous leukemia. Value Health. 2010;13(1):103-11.

28. Tufts cost effectiveness analysis registry. 2020. http://healthecon omics.tuftsmedicalcenter.org/cear4/SearchingtheCEARegistry/ SearchtheCEARegistry.aspx. Accessed Jan 2020.

29. Ara R. NICE DSU technical support document 12: the use of health state utility values in decision models. 2011. http://niced su.org.uk/wp-content/uploads/2016/03/TSD12-Utilities-in-model ling-FINAL.pdf. Accessed Jun 2020.

30. US Bureau of Labor Statistics. 2021. https://www.bls.gov/cpi. Accessed Apr 2021.

31. Rochau U, Kluibenschaedl M, Stenehjem D, et al. Effectiveness and cost-effectiveness of sequential treatment of patients with chronic myeloid leukemia in the United States: a decision analysis. Leuk Res Treat. 2015;2015:982395.

32. Reed SD, Anstrom KJ, Ludmer JA, Glendenning GA, Schulman KA. Cost-effectiveness of imatinib versus interferon-alpha plus low-dose cytarabine for patients with newly diagnosed chronicphase chronic myeloid leukemia. Cancer. 2004;101(11):2574-83.

33. Fenwick E, Marshall DA, Levy AR, Nichol G. Using and interpreting cost-effectiveness acceptability curves: an example using data from a trial of management strategies for atrial fibrillation. BMC Health Serv Res. 2006;6:52.

34. Cortes JE, Kim DW, Pinilla-Ibarz J, et al. A phase 2 trial of ponatinib in Philadelphia chromosome-positive leukemias. N Engl J Med. 2013;369(19):1783-96.

35. Latimer N. NICE DSU Technical Support Document 14: Survival Analysis for Economic Evaluations alongside Clinical TrialsExtrapolation with Patient-Level Data. Report by The Decisions Support Unit; 2011.

36. Marseille E, Larson B, et al. Thresholds for the cost-effectiveness of interventions: alternative approaches. Bull World Health Organ Policy Pract. 2015;93:118-24.

37. Dubois RW. Cost-effectiveness thresholds in the USA: are they coming? Are they already here? J Comp Eff Res. 2015;5(1).

38. Ward T, Jones B, Harrison JP, et al. The clinical and cost effectiveness of Dasatinib versus Nilotinib for the first and second line treatment of people with chronic myeloid Leukaemia. Value Health. 2017;20(9):A433.

39. Wu B, Liu M, Li T, et al. An economic analysis of high-dose imatinib, dasatinib, and nilotinib for imatinib-resistant chronic phase chronic myeloid leukemia in China. Medicine (Baltimore). 2017;96(29):7445.

40. Hoyle M, Rogers G, Moxham T, et al. Cost-effectiveness of Dasatinib and Nilotinib for Imatinib-resistant or -intolerant chronic phase chronic myeloid leukemia. Value Health. 2011;14(8):1057-67.

41. Vemer P, Ramos IC, van Voorn GAK, et al. AdViSHE: a validation-assessment tool of health-economic models for decision makers and model users. Pharmacoeconomics. 2016;34:349-61. 